\title{
Aplicación de estrategias didácticas para el desarrollo del pensamiento numérico en los estudiantes de octavo de la institución educativa nuestra señora de las mercedes en el municipio de Sardinata
}

\section{Application of didactic strategies for the development of numerical thinking in eighth- grade students of the Nuestra Señora de las Mercedes educational institution in the municipality of Sardinata}

\author{
Maribel Escalante-Albarracín ${ }^{\mathrm{a}}$ \\ a* ${ }^{*}$ Magister en educación, Institución Educativa Nuestra Señora de las Mercedes, Código Orcid 0000-0001-5749-7423, escalanteamaribel29@gmail. \\ com, Código Orcid 0000-0001-5749-7423 Institución Educativa Nuestra Señora de las Mercedes, Sardinata, Colombia
}

Forma de citar: Escalante-Albarracín, M. Aplicación de estrategias didácticas para el desarrollo del pensamiento numérico en los estudiantes de octavo de la institución educativa nuestra señora de las mercedes en el municipio de Sardinata. Eco Matemático, 10 (2), 16-22

Recibido: 5 febrero 2019

Aceptado: 9 abril 2019

\section{Palabras clave \\ expresiones algebraicas, estrategias didácticas, pensamiento numérico.}

\begin{abstract}
Resumen: La presente investigación tuvo como objetivo general diseñar herramientas didácticas para el desarrollo del pensamiento numérico en los estudiantes del grado octavo de la Institución Educativa Nuestra Señora de las Mercedes, Municipio de Sardinata. De acuerdo con los objetivos del estudio la metodología presenció un proyecto factible, ubicado en un nivel descriptivo, apoyado en una investigación de campo. La población escogida fue el grado Octavo de la Institución Educativa Nuestra Señora de las Mercedes, municipio de Sardinata los cuales constituyen un número de 125 estudiantes. Por otra parte la confiabilidad de los instrumentos de medición se determinó mediante el método estadístico Alpha de Cronbach y los datos se representaron y analizaron bajo la construcción de tablas de frecuencia y gráficos porcentuales, arrojó que los estudiantes poseen escaso dominio del pensamiento numérico porque no se encuentran motivados hacia el desarrollo del mismo, por otra parte los docentes deben implementar estrategias para que los educandos muestren interés por su aprendizaje de allí la necesidad de generar como estrategia didáctica el uso del juego que sirva como estrategia didáctica para el desarrollo del pensamiento numérico.
\end{abstract}

\section{Keywords}

algebraic expressions, didactic strategies, numerical thought.

\begin{abstract}
The present research had the general objective of designing didactic tools for the development of numerical thinking in the eighth-grade students of the Nuestra Señora de las Mercedes Educational Institution, Municipality of Sardinata. Following the objectives of the study, the methodology witnessed a feasible project, located at a descriptive level, supported by field research. The chosen population was the eighthgraders students of the Nuestra Señora de las Mercedes Educational Institution, Sardinata municipality, which constitutes a sample of 125 students. Otherwise, the reliability of the measurement instruments was determined using cronbach's alpha statistical method and the data were represented and analyzed under the construction of
\end{abstract}

*Autor para correspondencia escalanteamaribel29@gmail.com

http://dx.doi.org/10.1016/j.eq/

2590-9215@ 2017 Universidad Francisco de Paula Santander. Este es un artículo bajo la licencia CCBYNCND 
frequency tables and percentage graphs, showing that the students have little command of numerical thinking because they are not motivated towards the development of it; on the other hand, teachers must implement strategies so students show interest in their learning, hence the need to generate as a didactic strategy the use of games which serve as a didactic strategy for the development of numerical thinking.

\section{Introducción}

El pensamiento numérico hace parte de los cinco pensamientos de las matemáticas (Villarroel V, 2009). Para el Ministerio de Educación Nacional, los sistemas numéricos deben tener como base este tipo de pensamiento (Obando Z \& Vasquez L, 2008). Desde una perspectiva general, puede definirse como la capacidad que tiene una persona de aplicar los conocimientos sobre los números y sus operaciones para resolver problemas de su cotidianidad, a partir de estrategias, modelos y algoritmos (Obando Z \& Vasquez L, 2008). El pensamiento numérico involucra la aplicación de criterios, juicios, autorregulación e interpretación para dar múltiples soluciones con respecto a una misma situación (Mcintosh, Reys, \& Reys, 1992).

Las habilidades anteriores, comprenden la interacción tanto de conceptos como de la misma cognición humana (Obando Z \& Vasquez L, 2008), que se logra con el paso del tiempo. A medida que el estudiante va creciendo, su pensamiento numérico es desarrollado. Otro de los aspectos importantes, es el entorno social y educativo en el cual se encuentra, ya que para que el estudiante razone, interprete y proponga soluciones de forma crítica, debe estar expuesto a diversas situaciones que sean significativas y contextualizadas (Obando Z \& Vasquez L, 2008). No es lo mismo que se hable de una suma corriente entre dos números, a que el estudiante se encuentre realizando una compra en un supermercado de dos artículos con los mismos números, que en la vida real serían representados en pesos.
Se ha visto que, en la educación tradicional, los estudiantes presentan poco desarrollo del pensamiento numérico, que puede ser asociado a deficiencias en situaciones como las mencionadas anteriormente. En el caso de los estudiantes de grado octavo, donde uno de los ejes temáticos es el aprendizaje de expresiones algebraicas, muchas veces presentan apatía con respecto al aprendizaje de estos temas, y hacen preguntas como la siguiente “¿y para qué nos sirve?”

Este tipo de cuestionamientos se hacen los estudiantes en todos los grados escolares con respecto a matemáticas, pues consideran que no encuentran la suficiente aplicabilidad en los temas, añadiendo la poca motivación y herramientas didácticas que dan como resultado un aprendizaje significativo deficiente (Aristizábal, Colorado, \& Gutiérrez, 2016).

Por otra parte, los estudiantes presentan diferencias en su progreso académico; algunos estudiantes aprenden de forma más rápida los conceptos y avanzan sin ningún tipo de problema.; otros tienen un ritmo más lento, pero sin ningún tipo de dificultades y otros estudiantes, presentan grandes falencias con respecto a su aprendizaje, por ejemplo, la memorización de las tablas de multiplicar y operaciones básicas (Usuga M, 2014).

Por estos motivos, se hace necesario crear estrategias didácticas enfocadas en desarrollar el pensamiento numérico, aplicarla a un grupo de estudiantes del grado octavo de la Institución educativa Nuestra Señora de las Mercedes en el municipio de Sardinata y hacer una evaluación

"las representaciones semióticas son a la vez representaciones conscientes y externas... permiten una mirada del objeto a través de la percepción de estímulos (puntos, trazos, caracteres, sonidos...) que tienen el valor de significantes" (Duval, 2017: 68). las figuras geométricas, los gráficos cartesianos, los esquemas, la escritura aritmética y algebraica, las tablas son algunas de las representaciones semióticas de mayor uso en la enseñanza y el aprendizaje de la geometría. 
cualitativa y cuantitativa acerca del nivel de desarrollo del pensamiento numérico que puedan tener los estudiantes.

\section{Materiales y métodos}

\section{Población y muestra}

Para la escogencia de la población se tuvo en cuenta principalmente dos criterios. El primero es, que la maestra investigadora pudiera aplicar sus instrumentos de evaluación y estrategias didácticas a cursos donde ella dictara matemáticas. Por otra parte, los estudiantes deberían ser de un curso donde ya tuvieran conocimientos en matemáticas con una dificultad mayor, para poder evaluar de una mejor manera el desarrollo de su pensamiento. Con los criterios anteriores, fueron escogidos los estudiantes del grado Octavo de la institución educativa Nuestra Señora de las Mercedes del municipio de Sardinata.

Teniendo en cuenta que, la investigación busca crear estrategias didácticas para el desarrollo del pensamiento numérico, esta obedece a un enfoque cuantitativo, debido a que se necesitan hacer mediciones y aplicar recursos que permitan evaluar si los estudiantes están desarrollando este pensamiento. Por otra parte, hay un aspecto cualitativo, ya que el profesor, se encarga de hacer observaciones y analizar desde su punto de vista el progreso que van teniendo sus estudiantes, conforme se va avanzando en la investigación. Sin embargo, esa información se puede cuantificar.

A partir del planteamiento del problema, el cual es el bajo desarrollo del pensamiento numérico en los estudiantes de octavo grado, que a su vez se ve reflejado en sus calificaciones de pruebas tanto externas como internas, se consideran una población de estudio importante, conformada por 125 estudiantes de la Institución Educativa Nuestra Señora de las Mercedes. La muestra empleada en la investigación es de tipo aleatoria, donde los estudiantes son escogidos al azar, con igual oportunidad de ser incluidos en la investigación.

\section{Instrumentos}

Antes de la aplicación de cualquier instrumento, es fundamental solicitar permiso al rector de la institución, junto con los padres y representantes de los estudiantes. Para esta investigación, se aplicará un pretest con el fin de verificar los conocimientos adquiridos de los estudiantes con respecto al pensamiento numérico (Aristizábal, Colorado, \& Gutiérrez, 2016). Los pretest consisten en una serie de preguntas objetivas, es decir, ejercicios relacionados específicamente con el tema de expresiones algebraicas. Este instrumento es muy útil porque es estructurado y permite tabular la información para tomar decisiones a partir de los resultados obtenidos.

El pretest en este caso consistió en 2 componentes. El primero, consta de 10 preguntas, relacionados con expresiones algébricas. Las preguntas tienen que ver con las expresiones para calcular distancias, representación de conjuntos de números, cálculos de perímetros de figuras planas y reducción de términos.

El Segundo componente, está relacionado con el clima escolar y las estrategias didácticas. Por ejemplo, cómo consideraban la explicación del profesor con respecto a la resolución de problemas, la planificación de sus clases, presentación personal, apariencia del salón de clases, actitud de sus compañeros y de los directivos de la institución. Además, se preguntó a los estudiantes si consideraban que el juego sería una buena estrategia para adquirir y aplicar sus conocimientos y si tenían algunas propuestas lúdicas para aplicar en las clases de matemáticas. Para este componente, se diseñaron un total de 15 preguntas, las cuales se muestran en la tabla 1 .

\section{Estrategias didácticas}

Las estrategias formuladas para el desarrollo del pensamiento numérico en los estudiantes del 
grado octavo de la Institución educativa Nuestra Señora de las Mercedes, se basan en juegos didácticos que la mayoría de las personas conocen. Esto es, dominó, lotería y barajas. Sin embargo, se adecuaron para que, a partir de las mismas reglas que tiene cada juego, se busque la adquisición de conocimiento sobre expresiones algebraicas, interpreten esa información y la apliquen para resolver un problema común como lo es en un juego de mesa.

\section{Dominó algebraico}

Es un juego de mesa que consiste en una fichas o baldosas rectangulares, que están divididas en dos partes (Reglamento, 2019). En el juego tradicional, cada mitad tiene un número. Sin embargo, en este caso, consiste en una expresión algebraica como un producto notable, o el resultado de una de ellas. El dominó tiene sus 28 fichas y la idea es que, la persona que gane es porque quedó con 0 fichas. En este caso se evalúa la capacidad que tenga el estudiante de conocer el producto notable, y la rapidez con la que pueda resolver dicha expresión. El dominó contiene varios ejemplos de productos notables con diferentes números y permite que jueguen máximo 4 personas.
Tabla 1. Preguntas relacionadas con clima escolar y estrategias didácticas. La respuesta debe ser sí o No. Fuente: Autor.

\begin{tabular}{cl}
\hline$N^{\circ}$ & \multicolumn{1}{c}{ PREGUNTA } \\
\hline 1 & $\begin{array}{l}\text { ¿Tus profesores promueven que los estudiantes se traten } \\
\text { con respeto? }\end{array}$ \\
2 & $\begin{array}{l}\text { ¿Se dialoga sobre las normas de convivencia con tus } \\
\text { profesores y compañeros? }\end{array}$ \\
3 & $\begin{array}{l}\text { Antes de que tus profesores desarrollen las clases ¿Te } \\
\text { preguntan qué sabes del tema que van a explicar? }\end{array}$ \\
4 & $\begin{array}{l}\text { ¿Tus docentes te orientan sobre lo que puedes hacer para } \\
\text { mejorar tu rendimiento? }\end{array}$ \\
5 & $\begin{array}{l}\text { ¿Estás satisfecho con el ambiente escolar que se } \\
\text { desarrolla en la Institución? }\end{array}$ \\
6 & $\begin{array}{l}\text { ¿Tus profesores te ayudan para que te des cuenta de qué } \\
\text { manera aprendes mejor? }\end{array}$ \\
7 & $\begin{array}{l}\text { ¿El medioambiente de aprendizaje en la Institución es } \\
\text { agradable? } \\
8\end{array}$ \\
¿Crees que el docente debe planificar otras estrategias \\
en la enseñanza de la matemática? \\
¿Te gustaría que el docente diseñará estrategias \\
didácticas, como apoyo para la enseñanza de la \\
matemática? \\
10
\end{tabular}

\section{Lotería expresiva}

La lotería tradicional, consiste en una serie de fichas que tienen imágenes duplicadas. Se ponen boca abajo, y el encargado de destaparlas, la lee nombrando la figura que aparece en ella. Quien la tenga en su tabla, la reclama y la coloca en esta. El primero que llene su cartón gana el juego (SCRIBD, 2019). En el caso de las expresiones algebraicas, el estudiante encargado de leer las fichas tiene en su poder el resultado de diferentes expresiones algebraicas, y los demás estudiantes tienen su cartón con la expresión algebraica. Ese cartón se va llenando conforme el estudiante va sabiendo el resultado de cada una de ellas. El primero en llenar su tablero, gana el juego. El número máximo de estudiantes para este juego son hasta 5 personas. 


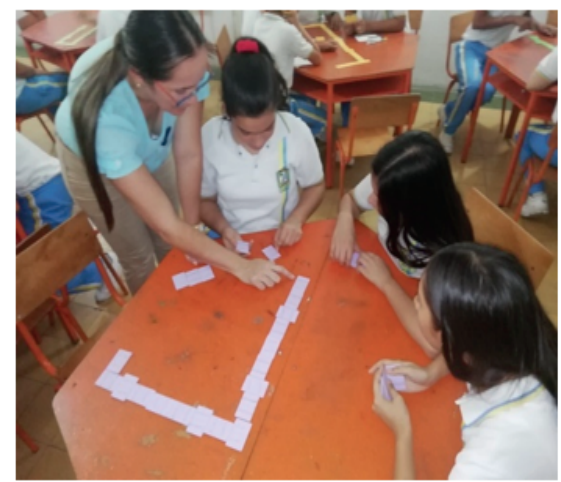

Figura 1. Aplicación del dominó de expresiones algebraicas en los estudiantes de octavo.

\section{Barajas de expresiones}

Este juego consiste en 48 cartas, para cuatro alumnos. El profesor se va a quedar con las cartas que tienen los resultados de las diferentes expresiones. Se reparten el resto de las cartas entre los alumnos. A continuación, se muestra una carta con un resultado y se invita a los estudiantes que tengan la expresión algebraica que da como resultado el número de la carta mostrada, colocarla junto a la suya. La idea es que, se formen filas de expresiones algebraicas que tengan el mismo resultado. Gana el estudiante que se quede primero sin cartas.

Después de aplicar las estrategias didácticas, se realizarán postest que contendrán una serie de preguntas similares a las hechas en el pretest para evaluar las herramientas y analizar el impacto que tuvo la nueva metodología. A partir de estos resultados, se evaluará si el desarrollo del pensamiento numérico aumento, disminuyó o se mantuvo igual para cada estudiante.

\section{Resultados y análisis}

\section{Resultados Pretest.}

El pretest fue aplicado a una muestra significativa correspondiente a 60 estudiantes, escogidos al azar. El pretest está compuesto por dos partes. La primera, son preguntas relacionadas con situaciones de la vida real donde deben aplicarse conocimientos y operaciones fundamentales de matemáticas, específicamente con el tema de expresiones algebraicas. Los resultados se muestran en la gráfica 1.

La segunda parte del pretest está relacionada con el clima escolar y las estrategias didácticas. Los resultados se muestran en la gráfica 2 .

Con respecto al primer componente, las respuestas permitieron obtener una conclusión unánime: Los estudiantes de octavo presentan altas deficiencias en el manejo de operaciones básicas y expresiones algebraicas y su aplicación a problemas de la vida real. La tabulación muestra que los estudiantes presentan altas deficiencias en cálculos de perímetros y áreas usando las expresiones algebraicas. Por otra parte, en siete de las diez preguntas, la mayoría de los estudiantes contestaron de forma incorrecta. En dos preguntas, la mayoría de los estudiantes tuvieron la respuesta correcta, y en una sola pregunta, el 50\% contestó bien y el otro $50 \%$ de forma incorrecta.

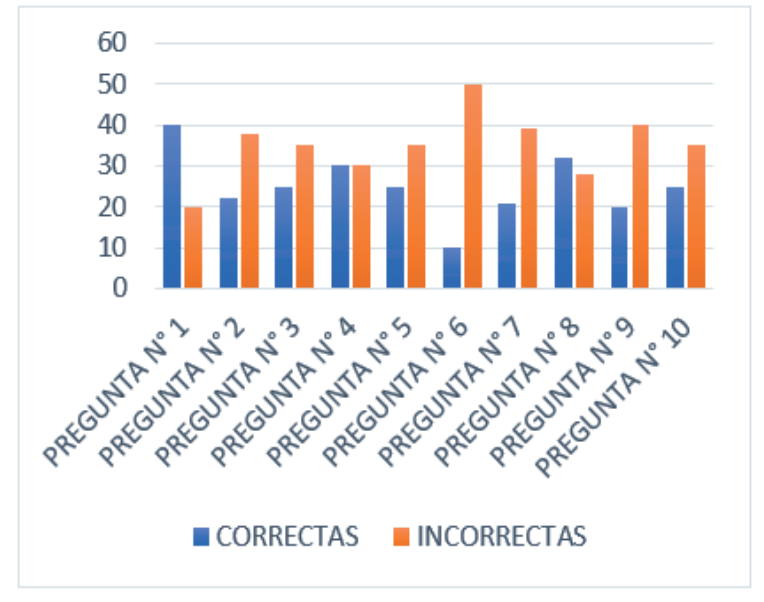

Gráfica 1. Tabulación de las preguntas sobre expresiones algebraicas en 60 estudiantes del grado octavo.

En cuanto al segundo componente, la maestra investigadora encontró respuestas positivas con respecto al clima escolar. Sin embargo, consideran que los espacios del salón y algunos horarios no son los más atractivos para ver las clases. En cuanto a las estrategias didácticas, los estudiantes 
en una decisión prácticamente unánime consideran que el juego es una estrategia atractiva para ellos, especialmente en matemáticas. Dentro de las propuestas se encontraron juegos como el dominó, parqués y cartas.

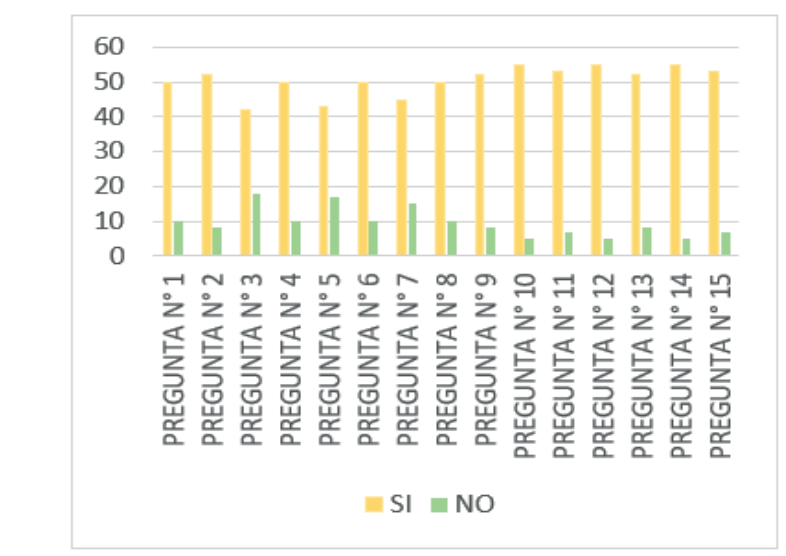

Gráfica 2. Tabulación de las preguntas sobre clima escolar y estrategias didácticas en 60 estudiantes del grado octavo.

\section{Aplicación de las estrategias.}

Antes de aplicar cualquier juego, se hizo la respectiva explicación del tema expresiones algebraicas y los subtemas asociados a este, pues es uno de los ejes temáticos más importantes en el grado octavo y sobre el cual se crearon los juegos. A partir de la explicación, se agrupan los estudiantes, de modo que los juegos puedan ser usados por todos.

Durante el juego, se puede ver que los estudiantes se deben volver ágiles, porque lo que se busca es que den una respuesta rápida para poder avanzar en el juego, y finalmente ser el ganador de la partida. Una de las observaciones claras que tiene la maestra investigadora, es la actitud con la que responden los estudiantes a este tipo de actividades. La motivación es alta y el espíritu competitivo hace que todos estén concentrados para no perder de vista ningún detalle y poder ganar. Como todo juego, hay un solo ganador, pero no es impedimento para que cada uno de ellos tenga un aprendizaje significativo con respecto a las expresiones algebraicos y además pueda desarrollar su pensamiento numérico de forma más dinámica y atractiva para ellos.

\section{Resultados Postest}

Para el Postest se adjuntaron preguntas muy similares a las aplicadas en el pretest. Esto es, con el fin de tener una comparación clara de los resultados antes y después de la aplicación de las estrategias. Nuevamente, se tienen los 2 componentes, pues para poder un aprendizaje significativo no solo es importante lo que pasa en el aula de clase, sino en toda su institución y la relación y presentación que tiene cada una de las personas que participa en su proceso de aprendizaje.

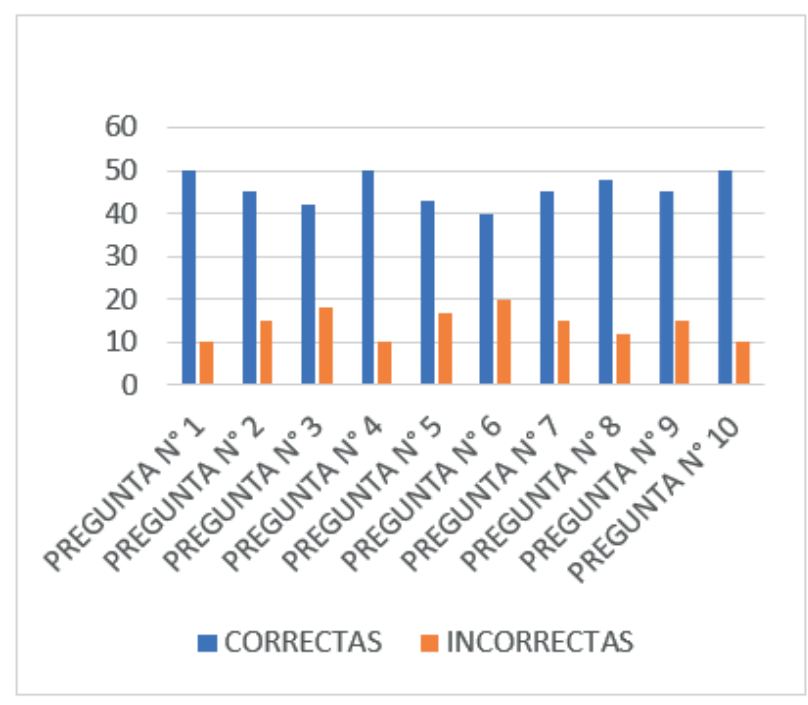

Gráfica 3. Tabulación de las preguntas sobre expresiones algebraicas hechas en el postest en 60 estudiantes del grado octavo.

Claramente, se ve una mejoría en la exactitud de las respuestas sobre el primer componente.

Los resultados se muestran en la figura 3 . Con respecto al segundo, sobre clima escolar, los estudiantes expresaron un cambio en el ambiente de su salón de clase y su relación con los demás compañeros. La motivación por aprender aumentó significativamente. En cuanto a estrategias significativas, propusieron otros juegos como Monopoly, Cartas, rompecabezas, entro otros durante la aplicación de las estrategias. 


\section{Conclusiones}

Para que el pensamiento numérico sea desarrollado en los estudiantes, es indispensable tener los conceptos de los diferentes procesos, número y operaciones matemáticas. Al tener claridad con respecto a lo anterior, y con un contexto real, el estudiante tendrá la capacidad de tomar decisiones en la resolución de problemas motivado por el hecho de que son situaciones que si pueden estar presentes en su vida.

Se evidencia que el juego, como estrategia didáctica para el aprendizaje, es una gran herramienta que permite a estudiantes y maestros lograr una relación más cercana con el conocimiento y a su vez desarrollar habilidades para la interpretación, inferencia y solución a diversos problemas.

La necesidad de cambiar las estrategias para el aprendizaje en el aula es evidente. La motivación y el interés por los estudiantes con respecto a un tema debe ser el objetivo general para cualquier maestro, no solamente en el área de matemáticas, sino también para las demás materias.

El fortalecimiento de las operaciones básicas en matemáticas es un trabajo de estudiantes, maestros y padres de familia, pues los alumnos a pesar de que avancen en sus grados, las deficiencias en estos temas se hacen más evidentes con el paso del tiempo.

Finalmente, se invita a que se realicen más investigaciones sobre estrategias didácticas, con el fin de fortalecer los diferentes pensamientos de las matemáticas, especialmente el numérico, que tanta utilidad presta para el desarrollo de las habilidades intelectuales de los estudiantes. Las investigaciones deben ser un trabajo conjunto de docentes y directivos docentes, con el fin de caracterizar muy bien a su población estudiantil, sus destrezas, habilidades y falencias que presentan en cada una de las materias, para atacar esos problemas y contribuir a un aprendizaje significativo integral en todos los estudiantes de la Institución educativa.

\section{Referencias}

Aristizábal, J. H., Colorado, H., \& Gutiérrez, H. (2016). El juego como una estrategia didáctica para desarrollar el pensamineto numérico en las cuatro operaciones básicas. Sophia, 2-12.

Mcintosh, A., Reys, B., \& Reys, R. (1992). A proposed framework for examining basic Number Sense . for the learning of Mathematics, 2-8.

Obando Z, G., \& Vasquez L, N. (2008). Pensamiento numérico del preescolar a la educación básica. Encuentro Colombiano de matemática Educativa, 1-21.

Reglamento, D. d. (22 de 11 de 2019). Reglamento del dominó. Obtenido de https://reglamentosdeportes.com/reglamento-de-domino/

SCRIBD. (15 de 10 de 2019). Obtenido de Reglas de Instrucción de Lotería y otros juegos : https:// es.scribd.com/document/384158027/Reglas-eInstrucciones-de-La-Loteria-y-Otros-Juegos

Usuga M, O. A. (2014). Diseño de una Unidad didáctica para la enseñanza-aprendizaje de la multiplicación de números naturales en el grado tercero de la Institución Educativa Antonio Derka Santo Domingo del municipio de Medellín. 2014: Universidad Nacional de Colombia Sede Medellín. Facultad de Ciencias. Villarroel V, J. D. (2009). Origen y desarrollo del pensamiento numérico: una perspectiva multidisciplinar. Electronic Journal of Research in Educational Psychology, 555-604. 